\title{
Penser les droits de l'homme avec Michel Villey
}

Michel Villey on human rights

Jean-Louis Vullierme

\section{OpenEdition}

Journals

Édition électronique

URL : https://journals.openedition.org/ress/4640

DOI : $10.4000 /$ ress.4640

ISSN : 1663-4446

\section{Éditeur}

Librairie Droz

\section{Édition imprimée}

Date de publication : 14 décembre 2018

Pagination : 241-259

ISSN : 0048-8046

\section{Référence électronique}

Jean-Louis Vullierme, «Penser les droits de l'homme avec Michel Villey », Revue européenne des

sciences sociales [En ligne], 56-2 | 2018, mis en ligne le 14 décembre 2021, consulté le 08 janvier 2022. URL : http://journals.openedition.org/ress/4640 ; DOI : https://doi.org/10.4000/ress.4640 


\title{
PENSER LES DROITS DE L'HOMME AVEC MICHEL VILLEY
}

\author{
JEAN-LOUIS VULLIERME
}

Paris, École normale supérieure

jlv@jlvnet.com

\begin{abstract}
Résumé: La critique de la notion de «droits de l'homme» de Michel Villey n'est pas conduite en opposition à l'esprit de justice, mais au nom d'une conception juridictionnelle (ou «réaliste») du droit. Le droit ne dérive pas de la volonté politique dite «souveraine» et des fondements métaphysiques dont elle se réclame (théologie, raison, morale, biologie ou sens de l'histoire), mais d'une multiplicité irréductible de sources. S'en suivent de graves objections relatives à l'inefficacité ou à l'inadaptation des formulations subjectives, volontaristes et législatives par lesquelles les «droits de l'homme» sont exprimés. Une reformulation juridiquement correcte ne serait pas purement nominale. Elle est d'autant plus nécessaire que le droit, rempart contre l'arbitraire, est menacé dans son essence même par les revendications du politique qui proclame les «droits de l'homme» sans les honorer, et à titre d'illusoire consolation pour ses possibles abus.
\end{abstract}

Mots-clés: droit subjectif, droits de l'homme, Michel Villey, philosophie du droit.

\begin{abstract}
Michel Villey's criticism of the notion of "human rights" is not carried out in opposition to a spirit of justice, but in the name of a judiciary (or "realist") understanding of law. Law does not derive from the so-called "sovereign" political will and the metaphysical foundations it alleges (theology, reason, morality, biology or the sense of history), but from an irreducible multiplicity of sources. There are serious objections to the inefficiency or inadequacy of the subjective, voluntarist, and legislative formulations used to express "human rights." A juridically adequate reformulation would not be purely nominal. It is all the more necessary since law, as a bulwark against arbitrariness, is threatened in its very essence by the demands of government, which celebrates "human rights" without fulfilling them, thereby providing an illusory consolation for its own possible abuses.
\end{abstract}

Keywords: human rights, Michel Villey, philosophy of law, subjective rights. 
Nous n'oublions pas que les droits de l'homme sont «opératoires»; qu'ils sont utiles aux avocats d'excellentes causes, protègent des abus du gouvernement et de l'arbitraire du «droit positif». Si par impossible on rayait ce terme de notre vocabulaire, encore faudrait-il le remplacer par un autre moins inadéquat. Nous ignorons lequel.

Tel est notre problème. (Villey, 2014 [1983], p. 14)

\section{INTRODUCTION}

Les droits de l'homme ont de nombreux faux amis qui pratiquent avec désinvolture l'hommage du vice à la vertu. Ils ont aussi de faux ennemis, ou ils en ont au moins un, qui ne les a critiqués que pour leur faible capacité à atteindre leurs objectifs de justice, sans pour autant contester ces derniers: Michel Villey n'est pas tant leur adversaire que celui d'une conception qu'il juge dangereuse du droit, et qu'il considère partagée tout à la fois par la plupart des partisans «progressistes» des droits de l'homme que par la plupart de leurs rivaux «réactionnaires». Ce qu'il prend pour cible est le «langage des droits de l'homme » en tant qu'il reflète de manière éclatante ce qui lui semble une incompréhension de la nature même du droit. Parce que le droit s'est, selon lui, constamment et fâcheusement déplacé de la juridiction au législateur, il devient outil d'ingénierie sociale au service du politique, en même temps que vaine proclamation formelle de garanties dont le justiciable peut se trouver simultanément privé. Il ne s'agit donc pas de revenir à un ordre social révolu, mais de mesurer le risque d'une dénaturation du droit sur laquelle les «proclamations des droits » jetteraient un voile pudique, alors même que le droit est, au bout du compte, la seule protection connue contre les tyrannies.

I Cette position est répétée p. 17 à propos des dissidents soviétiques. Ces propos ne peuvent être interprétés que d'une seule manière: si Michel Villey combat la conception du droit rattachée au concept de Droits de l'Homme, il en accepte expressément les visées de justice, tout en considérant qu'elles ne peuvent être atteintes par son moyen. 
Aux yeux du connaisseur de l'Antiquité gréco-latine qu'était Villey, la philosophie étant avant tout parole vive et échange dialogique évolutif, elle est susceptible d'engendrer de sérieux malentendus une fois fixée par l'écrit. Or ce sont surtout des cours et des notes, plus ou moins adaptés pour l'édition, que nous possédons de lui, tandis que le lieu matriciel de sa pensée, les dialogues, est demeuré non retranscrit. Le risque d'incompréhension s'est révélé bien réel dans son cas, d’autant qu'un style délibérément provocateur destiné à réveiller les esprits ${ }^{2}$, faisait perdre le fil des hésitations, des retours en arrière et possibles reformulations plus subtiles dont tout esprit honnête se doit de préserver la possibilité, comme il y veillait en réalité pour son propre compte. Le résultat a été à certains égards désastreux, ses disciples ${ }^{3}$ rivalisant parfois avec ses adversaires pour défigurer sa contribution. Je n’entrerai pas ici dans le détail de ces interprétations qui, pour l'essentiel, poussent ce catholique libéral dans un sens réactionnaire, anti-libéral, voire anti-démocratique ou théocratique, et cet admirateur de l'aptitude d'Aristote et de Thomas d'Aquin à opérer par synthèse de points de vue opposés, dans une direction obscurantiste et autoritaire avec laquelle il n'éprouvait aucune affinité. On en est même venu à identifier sa critique de la Modernité à quelque chose qui pourrait ressembler à ce qui lui répugne, qu'il s’agisse de Heidegger ou de Maurras

2 En relisant les œuvres de Villey, j'aperçois, trônant en exergue du Droit et les droits de l'homme, une citation de H. Mellic et l. Lévy dont je m'étais rendu coupable. II n'existe aucun «Journal of nuclear engineering», encore moins à «StanDford », et «H. Mellic et l. Lévy » forment une anagramme. Lorsqu'il en fit la découverte lors d'un colloque qui lui était consacré, la facétie étant une part intime de sa personnalité, sans laquelle il n'est pas toujours possible de comprendre ses textes, je fus promptement pardonné.

3 John Milbank en fournit un exemple remarquable. II se réclame de Villey et de sa critique des droits de l'homme, en faveur d'une organisation sociale fondée théocratiquement, une idée entièrement étrangère à la conception du droit de Villey. Voir Milbank, 20 I2, p. 203 et suiv.

4 Villey s'en prend vigoureusement à la «Nouvelle Droite»: «Le racisme, dont nous avons fait l'expérience en plein $x x^{e}$ siècle, est l'effet du positivisme, ou du scientisme des modernes [...]. Ses racines ne sont pas grecques. Et quant au racisme pris en charge par la Nouvelle Droite, il se recommande d'une pensée diamétralement opposée à la philosophie classique: du nominalisme qui a connu sa grande éclosion au début de l'époque moderne. Le nominalisme avait nié l'existence de "l'Homme". Et c'est une thèse très répandue au $x x^{e}$ siècle que la négation de la nature de l'homme, autour de Sartre, chez un grand nombre d'intellectuels. Tous n'ont pas été jusqu'à conduire à ses conséquences extrêmes. Mais ils voudraient qu'entre les 
Je ne chercherai pas non plus à justifier sa version de l'histoire de la philosophie juridique et de l'histoire du droit, ni dans son détail inévitablement fautif en regard des progrès de l'érudition ${ }^{5}$, ni même dans sa reconstruction d'ensemble qui a du reste varié avec le temps. Aucun philosophe n'est vraiment historien, et Villey pas plus qu'un autre. Mieux vaut considérer sa vision du droit antique comme un concept qu'il forge, plutôt qu'un récit minutieux ayant l'ambition de résister à la dissection savante. Ceci se manifeste au premier regard par le télescopage auquel il procède, parmi d'autres exemples, entre Cicéron et Gaius, Aristote et Thomas, dont il ne s'encombre pas toujours de mesurer les distances. Il s'agit plutôt d’exposer le principe de sa compréhension du droit, en tant qu’apport philosophique fécond.

\section{UN AVANT ET UN APRÈS MICHEL VILLEY}

Sur le plan du contexte, il faut bien voir qu'il y a, dans la philosophie du droit en France, un avant et un après Villey. Auparavant, la discipline, à la différence de l'histoire du droit, était mineure, souvent enseignée par des juristes autodidactes en philosophie et au seul usage des facultés de droit, tandis que régnait en maître la philosophie politique conduite au loin par des philo-

hommes seule fût commune la "liberté". De l'exercice de nos libertés naîtraient, s'ajoutant à celles qu'ont déjà reconnues des généticiens, de croissantes inégalités que la Nouvelle Droite contemple avec ravissement. 》 (Villey, 2014 [1983], p. 82 sq.). C'est correctement qu'il identifie les sciences sociales du XIX ${ }^{e}$ siècle comme l'une des sources essentielles du racisme ayant empoisonné le $x x^{e}$ siècle (voir Vullierme, 2018 [20।4], 2.2) et qu'il l'oppose au monde gréco-romain dans lequel la couleur de peau n'est effectivement pas un facteur de disparité politique. L'homme ancien est, d'après lui, une unité anthropologique que les modernes se sont employés à scinder en groupements rigides (d'où sans doute la référence à Sartre qui a défendu une conception ethniciste et communautariste des peuples décolonisés, par opposition aux universalismes de la pensée libérale ou soviétique). La notion de droits de l'homme servirait ainsi à recoller ce qui a été scindé, sous l'égide des états unitaires qui sont le produit même de la division. Villey n'est laudator temporis acti que sur des sujets limités. II est partisan de restreindre la notion de progrès aux sciences et techniques, persuadé qu'il était que les tyrannies du $x x^{e}$ siècle, qui se sont toutes exercées au nom du progrès, doivent se comprendre comme des régressions juridiques.

5 Pour quelques erreurs, en particulier sur la philosophie médiévale, voir par exemple (Piron, 2008). L'auteur conclut qu'il faut «donner congé» à Villey sur la base du caractère daté de ses connaissances historiographiques, sans peut être réaliser qu'à l'aulne de ce critère, il n'existe pas un seul philosophe dont il ne faille prendre congé. 
sophes n’ayant le plus souvent pas l'expérience des réalités juridiques. Ceux-ci s'étaient généralement persuadés d'en savoir l'essentiel à la seule lecture de Platon, Hobbes, Locke, Rousseau, Beccaria, Hegel et Kant, etc., parfois étendue à Hans Kelsen, Carl Schmitt et Léo Strauss, beaucoup plus rarement à Herbert Hart ou Ronald Dworkin.

Le Séminaire était alors fréquenté moins par des philosophes spécialisés $^{6}$, que par des mandarins des facultés de droit, venus d’abord y chercher un supplément d’âme philosophique. Ces éminents juristes, comme le civiliste Jean Carbonnier, sentaient bien que la «grande théorie» de philosophie politique ne correspondait pas à leur pratique, même et peut-être surtout quand le mot de droit y était prononcé. Villey est celui qui combla ce fossé. Il permettait enfin aux juristes de se rapporter à la conceptualisation philosophique d'une manière qui fît sens en droit, et aux philosophes de découvrir, souvent pour la première fois, d'authentiques objets juridiques qui ne fussent pas réductibles à la loi.

Il y a certains points communs entre ce que Villey pensait lui-même de son ouvre et la description qui en est faite par les auteurs qui par ailleurs la défigurent. D’abord l'excavation d'un «droit naturel classique », conforme par hypothèse à l'esprit du droit gréco-romain, entièrement recouvert par un «droit naturel moderne » pourtant antithétique, et ayant conduit une discipline entière, l'histoire du droit romain, au travestissement de son domaine. Ensuite la critique de la notion de «droit subjectif» identifiée comme étrangère à l'essence même du droit. Le droit subjectif, tel qu'il est pensé par Villey, consiste à imposer les volontés d'une puissance politique, au nom de justifications métaphysiques et par le canal législatif, tandis que le droit est, selon lui, foncièrement juridictionnel et «réaliste», au sens où la juridiction vise à dire le droit tel qu'il existe à la rencontre de la multiplicité de ses sources. On peut recenser

6 Je fus moi-même introduit à Villey par Louis Althusser qui entendait éloigner in partibus infidelium un chercheur perdu pour le marxisme, au grand dam de Jacques Derrida qui n'avait pas encore tenté d'investir le domaine sans le connaître.

$7 \quad$ Villey traite le droit gréco-romain comme un tout, non pour nier sa diversité, les oppositions qui le traversent, les singularités doctrinales de ses contributeurs, ses évolutions, ou interdire l'étude des particuliers, mais parce qu'il y reconnaît une unité transversale analogue à celle que discerne Paul Veyne lorsqu'il parle, plus largement encore, de «l'Empire-gréco-romain» (Veyne, 2005). 
bien d'autres aspects de son enseignement et de sa pensée, y compris moraux ou théologiques, mais je crois qu'il aurait à la rigueur accepté que l'on résume ainsi sa contribution, pour autant qu'elle ne soit pas davantage déformée.

Le mot de «réalisme » étant polysémique, il convient de préciser que c'est au sens étymologique que Villey entend l'employer : la res, la «chose», est ce qui nous concerne, ce qui est pour nous en question. Res publica ne signifie l’État que dans les mauvaises versions latines, mais désigne en fait ce qui, dans une communauté politique, concerne tout un chacun et doit donc y être discuté publiquement. Res possède ainsi une signification d’abord juridique, au sens de ce qui concerne des hommes entre eux, l'affaire, le litige, le cas $^{8}$. Quand l'affaire est soumise à un tribunal, elle devient causa. Ce n'est que par une dérivation ultérieure que ce qui advient et nous concerne devient la «cause» produisant «l'effet». D’après Villey, qui estime suivre la correction de Platon par Aristote, le concept («l'Idée») n’est pas premier. Il est un outil permettant (de manière procédurale, documentée et rationnelle), de comprendre et traiter, s'il y a lieu, ce qui advient dans le monde. Or, comme tout ce qui échoit est naturellement susceptible de produire un litige, le droit est l'organisation qui vise à sa résolution, en attribuant à chacun ce qui lui revient au vu de ce qui est advenu, si du moins une partie s'en plaint. Il établit et interprète les faits qui se sont produits, la situation, l'intentionnalité des acteurs, la justice ou l'injustice des conséquences; et ce, dans le respect des procédures et en mobilisant en tant que de besoin toutes les sources. Ce faisant, il est un processus régulateur naturel, propre à toute vie sociale qui ne serait pas régie par la seule violence. Sa disparition est une pathologie. C'est en ce sens que la conception juridictionnelle du droit peut être dite «réaliste», à partir de la conception gréco-romaine de la res. Elle s'oppose, par son caractère vivant, à une conception abstraite et statique, faisant du droit le produit d'une subjectivité volontaire et souveraine s'assignant d’autorité l'ingénierie d’une société. Si des lois ont été posées, le droit s’y réfère

8 Res ou chose qui concerne se dit en allemand Ding ou Sache, et en anglais thing. En vieil haut allemand, Dinc ou Ting signifie l'assemblée réunie pour résoudre un litige, une affaire en question, qui concerne à un titre ou un autre les personnes présentes. La même métonymie se rencontre dans les langues slaves. Voir Vullierme, 1979. 
évidemment, non sans vérifier leur applicabilité au cas, mais il peut encore se poursuivre en l'absence éventuelle de formulations législatives préalables, en utilisant d'autres sources. En aucun cas il ne faut confondre cette réalité naturelle du droit avec l'affirmation inverse que le droit est tenu par une «nature » conceptuellement prédéterminée de l'ordre social. C’est cette même erreur que Villey croit reconnaître chez les tenants du «droit naturel moderne», comme chez ceux du pur volontarisme politique.

Au moment où Villey prend la parole, une conception législative ${ }^{9}$ du droit l'emporte dans l'esprit des philosophes politiques français ${ }^{10}$. À leurs yeux la quintessence du droit est la «loi», entendue comme formule de loi édictée par un parlement, et elle est en principe le produit exclusif d'une «volonté souve-

9 Villey parle plus volontiers, et très correctement au point de vue juridique, de «droit positif» plutôt que de législation. Cette terminologie est cependant ambiguë pour un usage philosophique, puisqu'elle pourrait sembler rattacher principalement le droit positif au «positivisme» de type comtien, ce qui n'est évidemment pas le cas.

10 II s'agit de la quasi-totalité des auteurs de philosophie politique, de toutes orientations politiques (hormis certains marxistes), nourris en France par l'idée que la «souveraineté» et la «volonté» qui l'exprime sont la source première du droit, toutes les autres sources étant écartées ou subordonnées à la première. II n'était pas rare de rencontrer, au Séminaire de Philosophie du droit de Villey, des membres du Conseil Constitutionnel déclarant ignorer qu'il existât une autre conception possible du droit. Même ceux qui s'opposaient à l'affirmation radicale selon laquelle a «juridiquement tort celui qui est politiquement minoritaire» au Parlement imputaient encore les garanties permettant la protection des minorités à une volonté constituante formelle d'intégrer au «bloc de constitutionnalité » une pluralité d'autres normes formulaires (notamment la Déclaration des Droits de l'Homme). Jamais, un droit vivant premier n'était pensé par eux comme la condition de possibilité et la limitation de la volonté constituante elle-même.

Les juristes, quant à eux, se référaient à des théoriciens internes du droit, appartenant le plus souvent à des écoles positivistes (en France, Leon Duguit ou Maurice Hauriou principalement) ou jusnaturalistes modernes, et poursuivant leurs travaux à l'écart des philosophes qui généralement les dédaignaient. Leur connaissance de la pratique juridique leur interdisait d'adopter une conception législative pure qui aurait ignoré le rôle de l'interprétation et de la jurisprudence. Mais aucun d'eux n'échappait au «légicentrisme», y compris François Gény, spécialiste des techniques d'interprétation, et auteur très conscient du caractère inévitablement lacunaire des lois. Villey, qui l'a beaucoup critiqué (voit par exemple Villey, 1963), bien qu'il soit de tous les théoriciens du droit l'un des moins éloignés de ses positions, lui reprochait une vision constructiviste éclectique incapable de recentrer le droit autour de la juridiction ou plutôt du système juridictionnel qui interdit à la fois le volontarisme de la loi et le gouvernement des juges, deux versants à ses yeux d'un même subjectivisme. La relation entre les deux auteurs mériterait toutefois d'être réévaluée. 
raine ». Les juridictions sont ainsi ramenées à des appareils d'exécution des lois. Si bien que le droit apparaît tout entier comme une extension de la politique par d’autres moyens, ce qui permet supposément d'en faire sans résidus la théorie à partir des concepts canoniques de la philosophie politique. La Révolution française est simultanément élevée au rang d’Histoire sainte de la politique : chaque philosophe est tenu de se situer par rapport à elle, ou de l'être contre son gré. Ceux qui ne l'ont pas expressément embrassée sont réputés l’avoir rejetée au titre de la contre-révolution et des anti-lumières. Les autres sont discriminés en fonction de l'acceptation ou non de la Terreur, et de la volonté d’aller ou non plus loin que la grande Révolution dans des directions sociales ou nationales.

C'est précisément cette problématique que Villey entend rejeter d'un bloc, et c'est ce qui a été le moins compris de lui. Il ne se situe pas d'un côté ou de l'autre, ne cherchant pas le salut du côté de la Révolution ou de la ContreRévolution. S’il semble plus proche de Burke sur lequel il a écrit de manière laudative, ou s'il imagine parfois l'être, c'est parce qu'il croit discerner chez les auteurs britanniques attachés au common law, les restes d’un héritage précieux beaucoup plus ancien que les auteurs continentaux, tout obnubilés qu'ils sont par la loi formulaire, auraient fâcheusement dilapidé.

Au sortir de la Seconde Guerre mondiale, il lui semblait patent que la pensée politique moderne avait échoué dans le sang, toutes obédiences confondues. Elle a non seulement été incapable de prévenir les conflits les plus meurtriers de mémoire d'homme, les tyrannies les plus macabres et les plus abjectes, mais elle les a rendus possibles. Pour détruire le nazisme, on avait dû recourir à un bolchévisme qui n'était lui-même arrêté par aucune considération humanitaire. Le libéralisme, qui n'était pas exempt de crimes, ne disposait pas des instruments intellectuels capables de faire pièce à de telles monstruosités. Il n'était qu'à voir combien la construction législative des normes chez Hans Kelsen pouvait être balayée comme un fétu de paille par la désinvolture du premier Carl Schmitt venu, faux chrétien et faux juriste ${ }^{\mathrm{II}}$, et ouvrir la voie de la simple

II Villey conditionnait la dénomination de «chrétien» à l'adoption personnelle d'une attitude évangélique, tenant pour non-chrétien le simple rattachement formel aux institutions chrétiennes, en particulier quand ce rattachement était porteur d'antagonismes à l'égard des autres 
substitution par Hitler de la source même de la «volonté souveraine» et de la norme fondamentale. La branche utilitariste des alternatives modernes ne lui paraissait pas de bien meilleur aloi, puisqu’elle procédait également par le moyen législatif pour imposer le résultat de calculs étrangement conformes à certains intérêts particuliers. Le kantisme avait certes le rare mérite de ne pas préconiser une forme ou une autre d'horreur, mais il confondait selon lui, la morale et le droit, et autorisait en pratique le despotisme légal et «éclairé » de quiconque se réclamerait de la Raison.

\section{LA REDÉCOUVERTE PHILOSOPHIQUE DES SOURCES EXTRA-LÉGISLATIVES}

Il lui paraissait évident qu'ily avait un problème de fond avec le prométhéisme moderne et son instrumentalisation du droit. Thomiste par choix personnel dans une famille positiviste, il avait ainsi découvert indirectement Aristote puis les codifications romaines. La sentence, lue dans le Digeste, non ex regula ius sumatur, sed ex iure quod est regula fiat (Julius Paulus Prudentissimus, 533, 50.I7.I), fut son Eureka: ce n'est pas de la règle formulée que vient le droit mais c'est à partir du droit existant que la règle est faite. Preuve était donnée qu'existait bien une conception du droit radicalement distincte de la conception moderne, une conception qui admet la législation mais n'en fait pas son fondement. Son centre est la juridiction, non le législateur qui peut seulement contribuer au

confessions et des autres peuples. Alors que le bon Samaritain peut être chrétien, n'est pas chrétien, selon lui, celui qui abandonne le commandement d'amour universel. De ce point de vue, l'antagonisme de Schmitt lui paraît un christianisme de pure forme, seulement destiné à thématiser une identité historique particulière par rapport à un Ennemi confondu avec le reste du monde. De manière semblable, Villey réservait la dénomination de «juriste» au praticien du droit qui s'emploie à en respecter les principes, les visées (la restauration de l'état antérieur à la lésion) et les méthodes dialogiques. La familiarité de Schmitt avec le langage juridique et son aptitude à produire brillamment des textes juridiques, dans l'indifférence au caractère subalterne de la textualité juridique par rapport à ses conditions, comme la subordination qu'il opère du droit à l'autorité tyrannique, sont à ses yeux des signes très sûrs d'un abandon du droit au profit de son apparence. Pour ces motifs, Villey n'accorda jamais crédit aux inlassables tentatives de Julien Freund pour le convaincre d'un intérêt de Schmitt, pour la philosophie du droit, qui soit autre chose qu'une illustration de ce que le droit volontariste et législativiste avait malheureusement fini par produire durant la première moitié du $x x^{e}$ siècle. 
droit. Le juste n'est pas situé dans la volonté de l'empereur ou des dieux, même s'il importe d'en tenir tout à fait compte. Il n'est pas le seul produit d'une assemblée éventuellement exposée à l'hystérie comme à la combinaison des intérêts personnels de ses membres. Il n'est pas déductible de l'observation des animaux ou du comportement des hommes, bien qu'elle entre aussi en considération. Il n'est pas situé dans les convictions morales des juges, même si l'équité échappe aux magistrats injustes. Le juste est recherché contradictoirement dans la confrontation des parties au seul sujet de la cause faisant l'objet de la saisine, conformément aux procédures et aux garanties qu’elles maintiennent, y inclus l'enchevêtrement des juridictions qui modère le gouvernement arbitraire d'un magistrat. Le droit est dit par la décision de justice, à partir d'une multiplicité incompressible de sources incluant, non limitativement, les principes généraux sans lequel la juridiction ne peut pas fonctionner, telle la séparation du juge et des parties, la coutume, la jurisprudence, la logique, l'équité, la doctrine, les contrats et la législation. Le droit est donc «réel», dans les choses. Il résulte de l'interaction des sources et des acteurs du droit, au rang desquels il faut compter les justiciables eux-mêmes, dont le comportement anticipe les décisions de justice pour ne pas s'y heurter mais qui sont bien capables avec le temps de faire tomber une norme en désuétude. Cette conception du droit était si puissante qu'elle pouvait encore, dans certaines circonstances, survivre à un despote, le ténébreux Justinien ayant ainsi été le commanditaire de très importantes compilations ou codifications.

Elle n'obligeait pas à s'imaginer que l'autonomie native du droit signifiait une indépendance à l'égard du politique. Les juridictions sont des parties majeures de la politeia, et tous les acteurs de la politeia sans exception sont aussi des acteurs du droit. La législation positive est aussi une source du droit, à condition de ne pas devenir la seule. Mais de même qu'un gouvernement peut tyranniser la cité tout entière, il peut tyranniser le droit, réduire son périmètre, voire le faire disparaître. Car le droit est un organisme vivant, et c'est en ce sens primordial qu'il est «naturel», et non au sens où les normes qu'il serait supposément chargé de véhiculer seraient situées quelque part en dehors de lui, dans quelque introuvable «nature» immuable et métaphysique (rationnelle ou biologique, 
religieuse ou historiciste), comme l'affirme à l'inverse le droit prétendument «naturel» moderne. Le droit naît, se développe et meurt, et il peut aussi renaître quand on l'a étouffé. Ce qu'il ne peut pas faire, car ce serait contraire à sa nature, est de demeurer intangible et fixé. La question du conservatisme ou du progressisme dans les opinions des philosophes du droit apparaît ainsi totalement subalterne, car la seule question dont ils doivent perpétuellement répondre est : quavez-vous fait du droit, comment l’avez-vous préservé dans sa réalité même? Avez-vous été seulement un agent du Pape ou de l'Empereur ou d'un Comité? Avez-vous été d'abord le défenseur occulte des intérêts dont vous vivez ou l'outil inavoué des haines qui vous animent, au prétexte du droit que vous auriez seulement réduit à une fantomatique apparence? Le droit peut en effet aisément se restreindre à des atours qui ne sont préservés qu’au motif de la propagande, de l'autorité ou d'un banal ordonnancement administratif. En toute rigueur, il n'y a pas de droit nazi, mais un théâtre d'ombres.

Finalement, grâce au recentrage proposé, les juristes pouvaient reconnaître en philosophie des notions conformes à leur pratique quotidienne, eux qui consacrent tous leurs efforts à agir en vue de la juridiction, pour prévenir les litiges ou les résoudre, au service des justiciables ou des magistratures, par l'exercice des métiers du droit, la recherche ou l'enseignement. Villey se plaçait aux antipodes de constructions qui se montraient ignorantes des réalités juridiques, cherchant fréquemment à asservir le droit à des visées d'ingénierie sociale au nom de «l'Histoire» ou de la «Volonté», devenues manifestement fourriers de mort.

Parmi les non juristes, nourris qu'ils étaient de notions législatives, beaucoup ne comprenaient simplement pas de quoi il retournait. Ils concluaient que Villey était «contre» le progrès ou «contre» la souveraineté populaire. Il n’en était rien. Il estimait qu'écarter une problématique défectueuse interdit de se positionner ensuite à l'intérieur de son questionnement. À ses yeux, le fait que l'on soit à titre personnel conservateur comme lui-même ou progressiste comme nombre de ses collègues, n’entretient aucun rapport avec la réalité de ce qu'est ou non le droit. C'est uniquement une fois qu'il est correctement identifié à partir de la juridiction, qu'il devient possible d'observer le droit et ses transformations, puis de se prononcer sur l'état dans lequel il se trouve ou 
les directions qu'il devrait prendre. Villey considérait au surplus que le sociologisme qui régnait alors chez les théoriciens avait pour effet d'inverser les choses: le droit n'était pas un «reflet»d'une société qui se ferait sans lui, non plus qu'un obstacle inertiel à ses aspirations, il est une composante de la société, du moins quand il subsiste, qui ne peut ni ramener à lui toutes les autres composantes ni être ramené à elles.

L’incompréhension était renforcée par «l’idéalisme» philosophique de ce penseur réaliste. Villey savait que les hommes sont guidés par leurs représentations (selon une formulation qui n'est cependant pas la sienne), mais s'était convaincu de ce que toutes les idées intermédiaires, comme les idéologies, dépendent au bout du compte de conceptions du monde exprimées philosophiquement. D'où sa tentative, inévitablement imparfaite, mais à vocation pédagogique, pour replacer la totalité de l'histoire de la philosophie politique sur l'inopiné lit de Procuste d'une dualité entre le classique et le moderne, le réel et le subjectif, Aristote et Platon, Thomas et Ockham, etc. Il dressait de cette manière contre lui non seulement les multiples spécialistes heurtés par l'absence de nuance à propos de chacun des philosophes évoqués, mais aussi, de manière mécanique, tous ceux qui tenaient en faveur le nombre impressionnant des auteurs écartés, à commencer par la cohorte disparate des Modernes fédérés contre lui, en quelque sorte à sa propre demande.

Ce même principe idéaliste le portait à rattacher toute thématique à des fondamentaux philosophiques, ce qui ne pouvait aller sans quelque artifice. C'est ainsi que la question du droit subjectif est présentée à partir de considérations ontologiques, mais avec pour effet d'occulter quelques fois la réalité pragmatique à laquelle elle correspond dans le droit effectif. La notion de droit subjectif doit d'abord être comprise comme signifiant un «droit à» au lieu d'un «droit de» Villey dit que nul n’a jamais «droit à la maison» mais qu’il existe un «droit de la maison». Cette distinction lui paraît essentielle, parce que la maison n'est jamais le simple objet d'une relation entre elle et un sujet qui en revendiquerait l'usus et l'abusus au titre d'un droit de propriété. Une telle réclamation pure serait privée de sens car la maison est toujours prise dans un système de droit où sont impliquées bien d’autres personnes et considérations que le propriétaire et sa 
propriété : non seulement la puissance publique sous ses diverses personnalités, mais aussi les voisins, les passants, etc., presque ad infinitum. Si l'une quelconque de ces personnes affirme qu'elle est lésée par la perturbation de la réalité juridique de la maison, provoquée par un tiers à son détriment, alors il y a «cause » judiciaire, si du moins la plainte parait suffisamment fondée. Il appartient alors aux instances saisies, le cas échéant en chaîne, de suum cuique tribuere: attribuer à chacun ce qui lui revient. Les juridictions se prononcent à cet effet en vertu des sources du droit, dont la volonté législative ou a fortiori constituante n'est qu’une part. Elles peuvent constater par exemple qu’une loi est matériellement tombée en désuétude, ou que son application littérale aboutit à des conséquences illogiques ou iniques, ou qu'il y a eu collusion entre un juges et une partie, ou qu'une doctrine ou une jurisprudence déterminée est pertinente, etc.

À la lettre, il n’y a donc pas de droit subjectif dans le droit, et il ne peut pas y en avoir, mais uniquement des tentations intellectuelles périodiques pour les introduire, au moyen de formulations peu compatibles avec le fonctionnement naturel du droit, et provenant, selon Villey, d'une anthropologie philosophique déficiente, axée sur la puissance du sujet. Il importe d’apprécier à quel point ces deux affirmations sont dissociables, bien qu'il n’ait jamais pu ou voulu envisager la possibilité d'une dissociation. On peut pourtant parfaitement admettre avec lui qu'il existe une nature intrinsèquement réaliste du droit, et ne pas en conclure qu'elle dépend d’une gigantomachie philosophique primordiale. Ce point est spécialement significatif quand est abordée la problématique des droits de l'homme.

\section{LE FOND DE LA CRITIQUE DU CONCEPT}

Les droits de l'homme sont pour lui de simples formulations subjectives (droit du sujet à ceci ou cela) qui aboutissent à des leurres ${ }^{12}$. Par exemple, la proclamation d'un droit universel à l'éducation ou à la santé lui parait une affirmation au mieux vide et au pire cynique, ni la santé ni l'éducation n'étant jamais des objets

12 L'entreprise est définie comme n'ayant «trait qu'au langage, à la correction de ce langage. Le langage conditionne la pensée. Capitale est la dépendance de nos opinions relativement au langage [...]. C'est l'office de la philosophie d'être l'ordonnatrice du langage» (Villey, 2014 [1985], p. 17). 
qui peuvent être réclamés ou fournis in abstracto. L’interrogation juridique concrète sur le «doit à» la santé devrait pouvoir au moins aboutir à la question «qui paye quoi ?» dans le cadre du «droit de la santé», et ce, alors que nul ne pense raisonnablement quaucun gouvernement n'acquittera jamais la totalité des factures médicales de l'Homme ou même toute facture présentée par le petit groupe des hommes situés à des titres variés sur son territoire. La proclamation du «droit à» la santé présente le caractère d'être gratuite. Il s'agit seulement d'émettre des obligations nominales sans titre exécutoire, au mépris de ceux qui les acceptent en consolation de leurs espérances. À supposer que l'on parvienne à un «droit opposable à l'éducation », rien n'empêcherait de solder l'obligation ainsi créée par l'émission de titres scolaires aussi dévalués que la monnaie d'un État insolvable.

Or ce ne sont pas seulement «les droits sociaux de l'homme» qui sont en cause. Que signifie même le droit à l'égalité juridique, alors que le droit est toujours différent selon le statut des justiciables? L’un est dépositaire de l'autorité publique, l'autre non, l'un dispose d'une qualification l'autre non, l'un est mineur l'autre non, etc. L'égalité peut seulement signifier la non-intervention d'une discrimination non statutaire, telle que la couleur de peau ou la croyance religieuse. Que se passe-t-il quand la législation a introduit une discrimination raciale, venant au confort de jurisprudences racialistes, elle-même appuyée sur des habitus racistes, ce qui a été longtemps le cas notamment aux États-Unis? Il y faut un revirement jurisprudentiel, conforté ou non par un amendement constitutionnel. En y regardant de près, on constate que le vrai changement aura été un changement de la doctrine dominante relative à la disparité statutaire. Sans ce changement, aucune proclamation de droits subjectifs ne peut parvenir à amender la réalité juridique. De ce point de vue, les droits subjectifs, et les Droits de l'Homme en tant qu'ils s'expriment entièrement comme droits subjectifs, sont un «opium du peuple», et Villey parvient même à comprendre que les marxistes s’en soient défiés (voir par exemple, Villey, 2014 [1985]. p. I I).

Au reproche de futilité de la discussion du langage juridique «alors que d'autres se soucient (du moins en paroles) des pendaisons des Iraniens ou des noyades des Vietnamiens» (ibid.), il répond qu'une expression mal construite, «lorsque même on en fait usage au service de très justes causes, [...] les sert mal, pouvant aller jusqu'à se retourner contre elles (ibid., p. I8). 
Ce sont là des objections valides et assez profondes, de nature doublement «réaliste», susceptibles d'être entendues par des partisans des Droits de l'Homme s'ils sont aussi des esprits scrupuleux qui, sur la base d'une conception plus correcte du droit, souhaiteraient toujours se doter des outils adéquats pour réduire la misère et l'injustice du monde, un projet que Villey entendait pour sa part comme chrétien ${ }^{13}$.

Elles ne sont pas non plus des objections simplement techniques. Car si ce n'est pas le rôle du droit de bercer les peuples d'illusions, pas plus que de servir de courroie de transmission aux projets du Prince, l'une de ses fonctions objectives est de constituer de facto le dernier obstacle à l'arbitraire de la puissance. Parce qu'il est le dernier lieu où les intérêts et les vues se confrontent de façon littéralement civilisée, son absence ou son atrophie est la marque la plus sûre de la tyrannie. C'est un autre point qu'il convient d'accorder à Villey. Il est vrai que les Modernes ont concédé à l'arkhè le pas sur le nomos. Ils ont affirmé et cru que le partage de l'arkhè, du reste surestimé dans les républiques modernes qui sont toutes des oligarchies placées sous le contrôle du suffrage populaire, impliquait par elle-même la présence du nomos. Il n'en est rien. La préservation d'un droit digne de ce nom est la ligne de partage. Telle est en fait la grande leçon du $\mathrm{xx}^{\mathrm{e}}$ siècle $^{\mathrm{i}}$.

13 Villey tire de Thomas d'Aquin la séparation du droit et de la religion: «Le droit, sur lequel l'Évangile est muet» (ibid., p. II4). Les conséquences en sont importantes, car si le droit ne peut par sa nature traiter tous les aspects de la justice, cela n'implique nullement que n'existent pas d'autres aspects de la justice hors du droit: «Rien ne serait alors prévu au bénéfice des autres hommes? Bien sûr que si, mais cette besogne ne ressortira pas du droit» (ibid. p. I 15), mais à la morale éclairée ou non par la religion.

I4 La pointe de la critique philosophique des droits de l'homme par Villey porte sur la question de l'arkhè. Hobbes a été identifié par lui comme la source philosophique la plus directe de la subjectivisation massive du droit. Or, «le droit de l'homme» hobbésien est anarchiste dans la mesure où il se définit à la lettre comme «the Liberty each man hath to use of his own power, as he will himself, for the preservation of his own Nature ». II suit que, dans le but de le préserver de l'autodestruction, il faut et il suffit de priver l'homme de ses libertés et du droit pour lui imposer une monarchie qui s'exerce en son nom et sous des apparences juridiques. Autrement dit, l'inauguration de la pensée subjective du «droit de l'homme» est anti-démocratique, et Villey est convaincu que ce défaut natif est sans remède, sauf par un abandon complet de la conception subjective (voir ibid., ch. 9, passim). 


\section{CE QUI RESTE DE LA PENSÉE DE MICHEL VILLEY SUR LES DROITS DE L'HOMME}

Il est ainsi concevable de penser les Droits de l'Homme avec Villey, sans adopter pour autant sa «chronosophie » ou son anthropologie philosophique, mais au moins sa compréhension du droit. Alors et alors seulement, il est possible d'aller au-delà de lui dans la pensée du droit, afin de convertir l'aspiration à la justice en véritables objets juridiques indemnes des objections qu’il a soulevées.

L'obnubilation par la Révolution française présente toutes les difficultés associées à une situation limite. L'existence de recommencements juridiques radicaux paraît mettre gravement à mal l'idée réaliste du droit, puisqu'une constitution, donc la législation, y semble alors bel et bien placée à la source exclusive du droit. Mais il s'agit d'une apparence. Il faut d'abord considérer la notion hégélienne selon laquelle «aucune constitution n’a jamais été écrite», formule qui se réfère à la description aristotélicienne de la constitution, à la lettre la politeia tout entière, comme physiologie des institutions, et non pas comme texte de lois. Se pose alors immédiatement la question de la place réservée aux juridictions dans l'organisme politique qui a muté. C’est là qu'apparaît la bifurcation. Ou bien les juridictions sont détruites, c'est-àdire converties en fantômes de juridictions, fonctionnant en réalité comme simples comités d'exécutions sommaires et de spoliations; ou bien elles sont aussitôt réintroduites et contribuent ainsi d'emblée à réduire le périmètre de la tyrannie. La Révolution américaine est un exemple de mutation radicale dans lequel le droit, fonctionnant selon les principes du common law, est protégé de manière systématique et délibérée par les nouvelles institutions. La voie un moment ouverte en France est distincte, offrant par étapes une version aussi peu juridique qu'il se puisse de la notion de «constituante» et prenant prétexte de l'état de guerre pour anéantir la séparation des pouvoirs et les libertés initialement proclamées. Dans un ordre juridique digne de ce nom, il n'eût pas été concevable de réintroduire les discriminations statutaires dont la disparition même avait été érigée en principe: par exemple, si le statut nobiliaire est aboli, il est illogique et inique de condamner une personne en tant qu'elle relève par hérédité dudit statut. 
La question de l'état de guerre est d’autant plus décisive que, par la médiation de Schmitt (voir Monod, 2016 [2007]), une théorie de l'exception juridique permanente s'est propagée chez les adversaires les plus résolus, à droite comme à gauche, de l'ordre juridique républicain. L'ennemi libéral pratiquerait en permanence la distorsion du droit pour imposer ses intérêts, justifiant ainsi la pratique réciproque chez les révolutionnaires, qu’ils soient «naziphiles» comme Schmitt lui-même, ou communistes comme certains de ses disciples.

Or, si un ordre juridique peut, sous certaines conditions, admettre un état de guerre ou un état d'exception, il est certain que tout état de guerre ou tout état d'exception n'est pas compatible avec la préservation élémentaire du droit. Le droit romain en offre l'illustration. Le fonctionnement des institutions est temporairement modifié à l'ouverture des Portes de Janus, c'est-à-dire durant les guerres. Avait aussi été créée l'institution de la «Dictature», magistrature en tout point régulière, strictement encadrée par la loi. Si un membre du Sénat affirmait que la situation, pour une raison quelconque (militaire, économique, voire religieuse), ne pouvait être utilement traitée par les magistratures ordinaires, et proposait la nomination d'un dictateur (qui ne pouvait en aucun cas être lui-même), la Curie procédait à un vote. Si la nomination était approuvée, un dictateur était désigné pour une durée ne pouvant excéder six mois et à l'intérieur des limites d'une mission étroitement définie, dans l'idée que si le problème soulevé ne pouvait être traité en six mois, la dictature avait échoué et devenait elle-même un nouveau problème. Au dictateur était associé un «Maître de la Cavalerie» qu'il ne pouvait ni nommer ni démettre. Au terme de leur mission, les deux hommes étaient soumis à un quitus aux conséquences éventuellement fatales pour eux, s'ils ne parvenaient pas à l'obtenir. Dans le même esprit, la révolution que fut le Principat d'Auguste, mettant fin aux guerres civiles, s'effectua sans abolition d'aucune magistrature républicaine, ni suspension d’aucune juridiction, mais par la possibilité donnée au Prince de cumuler désormais une pluralité de magistratures. Le régime avait incontestablement changé, mais l'ordre juridique était maintenu, et pas seulement de manière nominale. Il devait perdurer longtemps, les dirigeants gothiques cherchant à y incorporer leurs coutumes sans altérer son ordonnancement ou abolir ses procédures. 
Il n'incombe certes par au droit de régir à lui seul les sociétés, mais sa présence trace une frontière. Elle est une condition sine qua non de l'exercice de libertés sans lesquelles toute perspective un tant soit peu analogue à celle des «droits de l'homme» devient aussitôt un non-sens. Reste à savoir de quelle manière «désubjectiviser» leur formulation, et donc leur exercice, sans renoncer à leurs objectifs. Sur ce point essentiel, le débat n’a pas encore eu lieu, les uns se contentant volontiers des formules existantes, en dépit des sérieuses difficultés qu’elles soulèvent, les autres les rejetant complètement sans autre forme de procès.

Sans prétendre le moins du monde régler ici d'un trait de plume cette grave question, il me semble que la réflexion doctrinale peut s'orienter dans la direction de l'interdiction des discriminations statutaires qui ne sont pas universellement et expressément admises, y compris dans l'administration des services publics, empêchant ainsi, de manière tout à fait pratique et contrôlable, la création subreptice de statuts d'exclusions. Inversant en quelque façon la perspective, il s'agirait de présumer devant les juridictions l'égalité statutaire quand elle n'est pas contredite par les sources du droit, plutôt que proclamer gratuitement de vains avantages sans en considérer les tenants et aboutissants. Ainsi les formulations subjectives du «droit à» pourraient faire une meilleure place à une universalisation des garanties associées à chaque «droit de», tout en écartant des formulations grandioses de «droits à» aussi aisées à saluer que malaisées à rendre effectives. Dans cette direction, nous aurions au moins contribué à penser avec Villey, sans pour autant solliciter son approbation tout au long de la route, mais en respectant sa profonde compréhension du droit.

NdA. Le présent article répond à une suggestion de Jean-Yves Pranchère et c'est à lui qu'il doit son titre. II résulte d'au moins sept années de dialogues directs avec Michel Villey, à partir de 1977, dans le cadre de son Séminaire de Philosophie du Droit de Paris II ou dans un contexte privé. 


\section{BIBLIOGRAPHIE}

LACROIX J. et PRANCHÈRE J.-Y., 2016 Le Procès des droits de l'homme, Paris, Éditions du Seuil.

JULIUS PAULUS PRUDENTISSIMUS, 533, Digeste, [en ligne]:

$<$ http://www.justinien.net>.

MILBANK J., 2012, «Against Human Rights: Liberty in the Western Tradition», Oxford Journal of Law and Religion, I-1, Oxford, Oxford University Press.

MONOD J.-Cl., 2016 (2007), Penser l'Ennemi, affronter l'exception, Paris, La Découverte.

PIRON S., 2008, «Congé à Villey», L'Atelier du Centre de recherches historique, I, [en ligne]: $<$ https://journals.openedition.org/acrh/3|4>.

VILLEY M., 1963, «Francois Gény et le droit naturel», Archives de Philosophie du droit, «Le dépassement du droit», 8, p. 12.

-, 2014 (1983) Le Droit et les droits de l'homme, Paris, PUF.

VEYNE P., 2005, L'Empire gréco-romain, Paris, Paris, Éditions du Seuil.

VULLIERME J.-L, 1979, «La chose (le bien) et la métaphysique», Archives de Philosophie du droit, «Les biens et les choses», 24, p.44-45.

-, 2018 (2014), Le Nazisme dans la civilisation: Miroir de l'Occident, Paris, L'Artilleur. 